\title{
Zur Persönlichkeit Karl Gustav Jungs
}

\author{
Von Friedrich Rintelen
}

Über das bewegte Leben und erfolgreich vielseitige Wirken Karl Gustav Jungs, des entscheidenden Reformators von Basels medizinischer Fakultät in der ersten Hälfte des 19. Jahrhunderts, ist Medizinalhistorikern wohl alles Wesentliche bekannt. Sein Ausbau des Anatomie-Unterrichtes, seine Leistungen als Internist, Pathologe und Allgemeinpraktiker, ungewöhnliches Organisationstalent, Einsatz für das gemeine Wohl für Stadt und Universität stehen im Vordergrund. Man ist manchmal versucht bei seinem Tun von multa eher als von einem multum zu sprechen; jedoch seine multa sind von ungewöhnlicher nützlicher Wirkung gewesen; und darum doch ein multum!

Doch wer war dieser 1795 in Mannheim Geborene wirklich, wie sein Charakter, sein Fühlen, Denken und Wollen? Stimmt das auch in dieser Hinsicht meist positive Urteil seiner Biographen, der Historiker, die sich mit Persönlichkeiten befaßt haben, die an Basels Hochschule zwischen dem Ausgang der Helvetik und Jungs Tod 1864 tätig waren? - etwa des älteren Wilhelm His, der bei der Eröffnung des Vesalianums in Basel 1875 betont hat, man verdanke ihm nicht nur die «Schaffung des anatomischen Institutes, sondern die Rettung der medizinischen Fakultät». Aber His täuscht sich, wenn er meint: «Bewunderswert bleibt bei Jung, dem Gemütsmenschen, sein steter Optimismus und unbeugsamer Lebensmut, der sich durch schwere Familiensorgen nicht brechen ließ.» Wenn der Chemiker Schönbein, ein Freund Jungs - sofern es das für den im Grunde einsamen Menschen überhaupt gab - am Grabe sagt: «Wo Jung war, da war Leben und Bewegung, Lust und Freude», so kann dies nur dem äußeren Erscheinungsbild gerecht werden. Edgar Bonjour findet von Jung: «er schien ein Sonnenkind des Lebens», spricht von seiner künstlerischen Veranlagung, er sei ein unkonventioneller Mann gewesen, der «nichts Steif-Professorales an sich hatte und gelegentlich im Übermut tollen Launen nachgeben konnte». Er sei «eine belebende Kraft geblieben, als schon dunkle Schatten sich auf sein Dasein senkten». Huldrych Koelbing, der sich oft mit Jung beschäftigt, hat 1954 in unübersehbarer Andeutung solcher «Schattenseiten» geschrieben: «Nach seiner Niederlassung in Basel ist Jungs Leben scheinbar ruhig und ohne große Veränderungen verlaufen; Kämpfe und persönliche Nöte, 
die er immer wieder zu bestehen hatte, ließ sich der liebenswürdige und umgängliche Mann offenbar kaum anmerken.»

Wenn man wissen möchte, wie es um Jungs Sosein bestellt gewesen ist, wenn man diesen Mann besser kennenlernen möchte, so tut man gut, sein höchst selbstkritisches Tagebuch durchzusehen; sein Sohn Ernst, der spätere Winterthurer Architekt, hat es 1911 herausgegeben. Vater Jungs Wunsch war es freilich, sein jüngster Sohn Paul, der Theologe, solle «alles in ein Heft schreiben, Einiges besser redigieren und seine Irrwege und Lasterwege - wie er sagt - für die Familie bewahren - nie aber aus den Händen lassen». Die «teilweise» Wiedergabe hat aber 47 Jahre nach Jungs Tod sein dannzumal einzig überlebender Sohn Ernst für angezeigt gehalten, es liege ihm daran, «Charakter und Denkungsart, von manchen seiner Mitmenschen, ja seiner Freunde mißkannt, zu Ehren zu bringen». Jung hat dieses Tagebuch, das er gelegentlich «Referat über den lumpigen Zustand meines Lebens» nennt, recht regelmäßig und eingehend von 1849 bis kurz vor seinem Tode 1864 geführt; oft hat er auf früher Erlebtes zurückgegriffen. Auf diese Blätter, die einen andern Jung zeigen, als den ihn die Öffentlichkeit kannte, werde ich mich in der Folge beziehen. Sie machen uns auch mit dem geradezu tragödienhaften Schicksal seines eigenen Familienkreises bekannt.

Jung ist dreimal verheiratet gewesen. Seine erste Frau, Virginie de l'Assault, die er noch in Paris kennengelernt hatte, starb 1828 nach 7jähriger Ehe. Von ihren 4 Töchtern starb die eine kurz nach der Geburt, zwei vernichtete $1845 \mathrm{im}$ «blühenden Alter» die Tuberkulose; die jüngste, Anna, das einzige Kind, das ihm offenbar nie Kummer gemacht hat, überlebte den Vater und war in der Ferne in Görlitz verheiratet. Jungs zweite Ehe mit der Württembergerin Elisabeth Reyenthaler dauerte 3 Jahre. Beide Kinder, die sie gebar, haben dem Vater fast nur große Sorgen bereitet. Der Sohn Karl, offenbar ein arger Nichtsnutz, wurde schließlich nach Amerika, in die «Europäische Besserungsanstalt», abgeschoben: «er soll von unten anfangen oder untergehen»; er ist dort verschollen. Die Tochter Caroline, eine «sündhafte Person» nennt er sie - tat ebenfalls nicht gut, lief von zu Hause fort, fing sich schließlich aber als Krankenschwester auf. Hat es dem vielbeschäftigten Vater an Zeit, an geduldigem Einfühlungsvermögen für die Schwierigkeiten seiner Kinder gefehlt, «fehlte es an Liebe, verlange ich zuviel?» fragt er sich selbstkritisch und sagt von sich: «Zu Hause bin ich ein König Leu, donnere, regiere und zanke» - man denkt an die Ätiologie heutiger Jugendunruhen. Jungs dritte Frau seit 1836, Sophie Frey, schenkt 
ihm - wohl eher nolens als volens - 5 Söhne, schließlich noch eine kleine Sophie. Die verlor ihre Mutter schon nach 3 Jahren durch eine Pneumonie. Der Sohn Rudolf, angehender Mediziner, ist 1857, Fritz 1863 an rasch progredienter Tuberkulose zugrunde gegangen; «an Schwindsucht, diesem Ungeheuer», sagt Jung. 9 Jahre lang hat der Witwer mit Max und Paul und dem kleinen «Hausfraueli», wie er Sophie schon als 3jährige nennt, zusammen gehaust. «Eine Kolonie des Jammers und Verlassenheit», findet er diese Gemeinschaft. Paul, der Theologe, ist der Vater Carl Gustav Jungs II, des Psychiaters und Psychologen. Wenn Jung von sich sagt, seine «Schwächen seien Ruhe, Besonnenheit, Maßhalten, Gerechtigkeit im Urteil», so mögen solche Mängel verständlich machen, daß er in der erzieherischen Führung seiner Kinder wenig Geschick zeigte. Warum findet man so häufig ehrgeizignegative Urteile über die Söhne: «die Holzböcke» - «keiner der Buben will nach; mittelmäßige Köpfe»! Aber eben: «ich will alles zu gut und gleich - er meint rasch - haben, da leidet meine Umgebung», und so kommt er zum Fazit: «bitter bin ich, oft habe ich meine Kräfte überschätzt, bin oft vom Ziele abgewichen».

Als Jung nicht ohne Widerstände 1822 als Professor für Anatomie, Chirurgie und Entbindungskunst nach Basel berufen wurde, lag schon viel Schweres hinter ihm, das sein späteres Leben beeinflußt und belastet hat. Seine Jugend im Mannheimer Elternhaus war keineswegs glücklich. Der Vater blieb ihm fremd, die geliebte Mutter, in deren Schoß er sich später gelegentlich zurücksehnt, neigte zu Depressionen - geistig sei sie zuletzt «gefesselt» gewesen. Jung spricht von seiner jammervollen Erziehung, er sei nicht zu Ordnung und Haushalten angehalten worden und habe «an einer von Jugend an eingeschlagenen Geringschätzung seines eigenen Wesens gelitten». Ein Martyrium nennt er einmal seine Jugend zurückblickend. Griechisch und Latein seien ihm geläufig gewesen, aber eine allgemeine Bildung des Geistes und des Herzens habe ihm gefehlt. Nach höchst erfolgreichem Heidelberger Studienabschluß - der vielseitig Begabte wäre fast Buchhändler geworden - folgte eine bewegte Zeit des Burschenschafters in Berlin. Unter Schleiermachers Einfluß tritt der Katholik Jung zum evangelischen Bekenntnis über.

Der Kampf des jungen stürmischen Drängers, der sich indes «übermäßiger Weichheit» des Gemütes beklagt, der Kampf des «Demagogen» gegen die Metternichsche Reaktion im Preußen Wilhelms III. bringt ihn für 13 Monate in Haft in der Berliner Hausvogtei und dann in die Verbannung nach Paris; das war schließlich sein Glück. Vor allem Alexander von Humboldt 
hat sich seiner angenommen und nach Basel empfohlen; «was er mir gewesen, wird in mir leben, solange ich lebe», sagt Jung anläßlich Humboldts Tod 1859. Trotz dem unerfreulichen Erlebnis in Preußen hat Jung an seiner deutschen Heimat gehangen. Immer wieder nimmt er lebhaft Anteil am dortigen politischen Geschehen. Da hört man Erstaunliches, wie: «wer an Deutschlands mächtiger Zukunft zweifelt, ist ein Rindvieh»; und fast fatal klingt sein Ausspruch: «Geduld, mein unglückliches Vaterland, Dein Messias ist geboren.» Hat er damit Bismarck gemeint? Eindeutig bekennt er sich zu einer Monarchie für Deutschland.

Bei all seinem Engagement für Basel - das er ein «Prosaisches Nest» nennt, ist er hier nie wirklich heimisch geworden. In einem Aufsatz über Heimweh spricht er vom «dulce natale solum». Und doch findet er: «ich bin kein Deutscher mehr»; mit der etwas eigenartigen Begründung: «dort kann ich nie mehr was sein». In Basel, «in einer Ecke der Welt, dem Vaterland gegenüber», wo man «kleinglücklich» lebe, findet er: «ich bin den Leuten nicht nach ihrem Maß»; er kommt sich als «Landesfremder» vor. Er, der offensichtlich Dank- und Lobbedürftige, leidet unter der für die Basler typischen Zurückhaltung im Aussprechen von Anerkennung. Dabei hat Jung «niemand dem ich mein Herz ausschütten kann, mir fehlt ein Freund; ich bin dafür geschaffen, allein zu sein». Es fehle ihm der Umgang mit Gleichgesinnten. «Warum bin ich so wenig verstanden»? «Ein Meer von Gleichgültigkeit umgibt uns!»

Im Juni 1822 ist Jung Ordinarius in Basel geworden. 2 Jahre später erwirbt er das Basler Bürgerrecht. Schon 1828 ist er Rektor der Universität, 11mal übernimmt er das Dekanat der medizinischen Fakultät. Bald sitzt er im Großen Stadtrat und ist Vorgesetzter der akademischen Zunft. Daß er jedenfalls zu Beginn seiner steilen Karriere eine innere Unsicherheit nicht hat ablegen können, zeigt sich, als er seine bekannte Schrift «Können in Basel die nötigen Hilfsanstalten zur Förderung medizinischer Studien gegründet werden?» anonym erscheinen läßt. Daß man im kritischen und sparsamen Basel das Vorprellen des Fremden, der behauptet, Basel habe die nötigen Mittel, um das Geforderte zu realisieren, nicht nur mit Wohlgefallen quittiert hat, ist naheliegend. Aber Jung setzt sich durch. Sein mutiger Einsatz als Zivilarzt während der blutigen Wirren um die Kantonstrennung im August 1833 hat ihm manche Sympathien eingetragen. 1835 ist die Basler Fakultät wieder zu einer propädeutischen «Pépinière» abgesunken; Jung wird auf halben Sold gesetzt und auf die Privatpraxis verwiesen. Er meint: «Ich hätte andernorts keine Praxis und Brot betteln nötig gehabt.» Es ist ein 
stetes Klagen über Geldsorgen, über die leere Kasse, über die «Arbeit für den verfluchten Brotkorb», was man von ihm hört - sie «vergälle sein wissenschaftliches Feld». Dabei gibt er gelegentlich große Gastierungen zu Hause, lebt vielleicht über seine Verhältnisse. Andeutungen einer manisch-depressiven Konstitution scheinen kaum zu übersehen.

Zu Gott, von dem in seinen Tageblättern fast auf jeder Seite die Rede ist, hat er eine schwer faßbare Beziehung. Himmelhoch jauchzend, zu Tode betrübt, ruft er ihn immer wieder fast kindlich an, Vertrauen bekundend, das er offenbar doch nicht recht besitzt. Gelegentlich ist ihm Gott, der «ewige Raum», man kann ihn nicht schauen, weil er überall ist. Ein eigentlich frommer Mensch, als den man ihn oft dargestellt hat, ist er wohl nicht gewesen. Dazu war Jung zu ich-bezogen, auch zu extravertiert. Sein gelegentlicher Ausruf: «nur keine ewige Seeligkeit», könnte bezeichnend sein.

Daß der «beliebteste Arzt Basel's», wie ihn His nennt, Wesentliches zum Entstehen des neuen Spitals an der Hebelstraße beigetragen, hat man hochgeschätzt, die menschliche Isolation des Spitalarztes und seit 1855 Ordinarius für praktische Medizin, hat sich deswegen nicht geändert; auch die Gründung der «Anstalt zur Hoffnung» vor dem Riehentor für behinderte Kinder scheint seine menschliche Isolation sowenig wie die Zugehörigkeit zur Freimaurerloge behoben zu haben. Ja, gerade seine Organisations- und Führungsqualitäten dürften den Aktiven, der es bis zum Großmeister der vereinigten Schweizerlogen (Alpina) gebracht hat, eher vereinsamt haben. Unter Geldsorgen und seiner steten äußeren Aktivität hat offensichtlich seine wissenschaftliche Produktivität gelitten.

Über Jung gäbe es noch viel zu sagen, was Verständnis für diesen Mann zu mehren geeignet wäre. Von seinen Mühsalen mit manchen physischen Bresten etwa, mit denen er fertig werden mußte. Vor allem hat ihn eine chronische Bronchitis geplagt, Husten, sein «Nachtwächter», der ihn gelegentlich zum Opium greifen ließ. 1853 hindern den 58jährigen «Augen und Bauch an Selbsthilfe bei der Pedicure».

Von seiner gelegentlich wechselnden Einstellung zur Musik, die seine Frau Sophie besonders pflegte, hätte die Rede sein können. Jedenfalls, die «Alceste», die große Oper Glucks, Beethovens 9. Symphonie und Mendelsohns Oratorium freuen und bewegen diesen musikalischen Menschen. Klara Schumann begeistert ihn mit Beethovens d-Moll-Sonate.

Die zugemessene Zeit verbietet, mehr als Lückenhaftes anzudeuten. Es ging mir darum, uns diesen K. G. Jung, dem Basel, seine Universität und die 
medizinische Fakultät Entscheidendes zu verdanken haben, in seiner Menschlichkeit näher zu bringen; ich meine dieser Mensch «in seinem Widerspruch» könne dadurch nur sympathischer werden.

Wenn Jung in sein Tagebuch bei Ende des Jahres 1854 einträgt: «ich bitte um gütige Nachsicht im Schlußurteil, wenn ihr mich nicht mehr haben werdet», so wollen wir ihm diese Bitte wohl gerne erfüllen.

\section{Summary}

If we want to consider the character of Karl Gustav Jung, who mainly reformed the medical faculty of the University of Basle in the first half of the 19th century, it is necessary to get acquainted with the diary that Jung has written between 1849 and 1864 . What we can state there, we have tried to show in this paper, especially in relation to his children. We see quite another man - not only the eminent organiser and many-sided practical and theorectical physician.

Prof. Dr. med. Friedrich Rintelen

Bruderholzrain 55

Ch-4059 Basel 